\title{
ESTUDIO DE LA USABILIDAD DE LA TECNOLOGIA DE INTERNET EN EL TURISMO RECEPTIVO EN LIMA-PERU
}

\section{USABILITY STUDY OF INTERNET TECHNOLOGY IN INBOUND TOURISM IN LIMA-PERU}

\begin{abstract}
Mery Morales ${ }^{1}$
RESUMEN

Estudios realizados en Ayacucho en el 2005, sobre las tecnologías de información aplicadas al sector turístico contemplan solo páginas web y correo electrónico. En este trabajo se centra el estudio en determinar la usabilidad del internet para captar turistas extranjeros y los problemas de su uso en las agencias mayoristas y minoristas. El estudio muestra que el nivel de usabilidad de la tecnología es la web, la cual es más informativo en ambos sectores, los servicios en línea solo registran pedidos, responden e-mail; sobre comercio electrónico las agencias mayoristas realizan muy poco canastas de pedidos y pago electrónico, en cambio la agencias minoristas realizan un mayor porcentaje de canasta de pedidos y pago electrónico; el personal de ambas agencias tienen un nivel de preparación muy adecuado, sobre el idioma más usado en las páginas web es el español; sobre los problemas de comercio electrónico que afectan el uso de las tecnologías son costos en las agencias mayoristas y procedimientos en las agencias minoristas.
\end{abstract}

Palabras clave.- Internet, turismo receptivo, Usabilidad, Tecnologías de información y comunicaciones.

\begin{abstract}
Studies done in Ayacucho in 2005, on information technologies applied to tourism websites and contemplate just email. This paper focuses on the study to determine the usability of the internet to attract foreign tourists and problems of its use in retail and wholesale agencies. The study shows that the level of usability of technology is: the web is more informative in both sectors, the services only register online orders, answer e-mail, e-commerce agencies made little baskets wholesale ordering and electronic payment instead the retail agencies perform a greater percentage of basket orders and electronic payments, staff from both agencies have a very adequate level of preparation on the most used language in web pages is Spanish on electronic commerce issues that affect the use of the technologies are cost wholesale agencies and retail agencies procedures.
\end{abstract}

Key words.- Internet, incoming tourism, Usability, Information and communications technologies.

\section{INTRODUCCIÓN}

El turismo es una de las industrias más representativas del país generando ingresos muy significativos alrededor del 3.7\% del PBI [14], y tenemos un rico legado cultural muy atractivo, pero nuestro nivel de visitantes no es el adecuado comparado con otros países europeos, sudamericanos, como Brasil. Una de las formas de promover el turismo es a través de las tecnologías, debemos conocer el uso de las mismas y medir el grado de usabilidad. El objetivo de la investigación es determinar la usabilidad de la tecnología internet, que ayudara a encontrar los problemas de

\footnotetext{
${ }^{1}$ Docente de la Facultad Ingeniería Industrial y de Sistemas de la Universidad Nacional de Ingeniería.
} 
uso y aplicación y; permitirá plantear soluciones tecnológicas que apoyen a incrementar el número de visitantes a nuestro país y que la industria de turismo crezca y sea competitiva en forma sostenida.

Existe un completo desconocimiento de las causas que impiden una gestión efectiva de la tecnología internet para captar más turistas extranjeros. Solo se usa internet para comunicarse por email y consultar información mediante buscadores; desaprovechando las facilidades de interacción en línea y registros en base de datos. Los índices de captación en turismo receptivo por internet son bajos comparados con los países de Centroamérica y sudamerica.

Los estudios realizados en al año 2005 [6], muestran que las tecnologías de información que se han aplicado al turismo peruano son el portal Go2peru.COM, Siturismo, Piuranet, Amadeus Perú, e-ticket.

El uso de las tecnologías de información en las agencias turísticas de Ayacucho, muestran que el $34 \%$ tienen página web, $27 \%$ acceso a internet, $38.6 \%$ correo electrónico, $25 \%$ realizan comercio electrónico.

Los problemas encontrados para vender por internet fueron: los productos no son adecuados, los clientes no están preparados, la seguridad, incertidumbre el marco legal, y problemas logísticos.

Las pequeñas empresas son las que tienen mayor déficit tecnológico.

El artículo ha sido organizado en 6 secciones, en la sección 2 son los conceptos referentes a las tecnologías de información, en particular el internet; sobre turismo y el nivel de usabilidad; en la sección 3, el estado del arte de investigadores recientes. En la sección 4, explicamos el método de investigación empleado; en la sección 5 mostramos los resultados de la investigación; primero a nivel grupal (agencias mayoristasagencias minoristas), segundo a nivel agencias mayoristas y tercero a nivel agencias minoristas. Finalmente la sección 6 arribando a las conclusiones sobre el uso de las tecnologías de internet de las agencias.

\section{TIC`S, TURISMO, USABILIDAD}

\section{Tecnología información y comunicaciones}

Las Tecnologías de la Información y la Comunicación, también conocidas como TIC, son el conjunto de tecnologías desarrolladas para gestionar información y enviarla de un lugar a otro. Abarcan un abanico de soluciones muy amplio. Incluyen las tecnologías para almacenar información y recuperarla después, enviar y recibir información de un sitio a otro, o procesar información para poder calcular resultados y elaborar informes.

Según Luana 2007 [10], las Tic`s que apoyan al turismo son:

Sistemas globales de distribución (GDS).Auténticas ventanillas únicas del turismo internacional.

El GDS Amadeus3.- Cuenta en la actualidad, con casi 150.000 terminales instalados en empresas turísticas de todo el mundo.

Los PMS.- (Property Management System) o sistemas de gestión de la propiedad, para la gestión hotelera.

El Internet.- Les permite realizar los procesos de marketing, desarrollar la imagen de marca, y crear productos virtuales. Las PYMES $(95 \%$ de las empresas turísticas) pueden ocupar nichos del mercado para alcanzar un mayor porcentaje de su grupo destinatario y de expandir su radio de alcance.

E-commerce.- De las diferentes modalidades de comercio electrónico existentes hasta el momento las empresas turísticas han tenido que recurrir en mayor medida al B2C (de empresa a consumidor) y el B2B (de empresa a empresa).

E-Turismo.- 2004 [15], el internet, intranets, extranet son herramientas claves para su desarrollo; además el e-Turismo, supone la virtualización de todos los procesos de gestión de la empresa y de la cadena de valor en el turismo, y tiene como objetivo maximizar la eficiencia de las empresas. Este nuevo concepto incluye a las funciones del negocio junto con la estrategia, la planificación y la gestión electrónica der los sectores que componen la industria turística. Negocio.- (Producción, marketing, finanzas), SI/TI (sistemas de información y tecnologías de información). 
Turismo.- (Proveedores de transporte, proveedores de alojamiento, proveedores de ocio, agentes de viaje).

Una innovación es Robotiker 2006 [11], centro tecnológico especializado en tecnologías de la información y de las telecomunicaciones, que ha desarrollado un sistema basado en tecnología GPRS-UMTS para la provisión de servicios turísticos. El objetivo del proyecto es dotar a los turistas de guías de viajes personalizadas. El sistema permite visualizar rutas predefinidas, proporcionar información de puntos de interés, ayudar al turista en sus desplazamientos por la ciudad.

\section{Turismo}

Según la OMT, 2008 [13] el turismo comprende las actividades que realizan las personas durante sus viajes y estancias en lugares distintos al de su entorno habitual, por un período consecutivo inferior a un año y mayor a un día, con fines de ocio, por negocios o por otros motivos. La OMT señala diferentes formas de turismo: i) turismo doméstico, se refiere a residentes visitando su propio país; ii) turismo receptivo, se refiere a no residentes procedentes de un país determinado; y iii) turismo emisor, que son los residentes del propio país que se dirigen a otros países.

Los productos turísticos son el turismo individual, masas, cultural, natural, activo, de negocios, científico, espacial. Los servicios turísticos es la prestación de servicios de alojamiento, alimentación, guía, acogida, información.

El turismo tiene efectos sobre la economía y la cultura en un país o región; los flujos económicos debido al turismo afectan tanto en términos macroeconómicos como microeconómicos tanto en las zonas emisoras como en las receptoras. Las repercusiones económicas del turismo se clasifican en: dependencia de la situación económica general, empleo, producción, distribución de la renta, tasa de cambio, inversiones públicas.

En los efectos culturales tenemos: alteración de las estructuras sociales, aculturación, mejor conocimiento de otras sociedades y mentalidades, estrecha los lazos de entendimiento entre estados, favorece el aprendizaje de idiomas extranjeros, efectos de las actividades artesanales, y efectos sociopolíticos.

Walter Hunziker - Kurt Krapf, 1942 [18], el turismo es el conjunto de relaciones y fenómenos producidos por el desplazamiento y permanencia de personas fuera de su domicilio, en tanto que dichos desplazamientos y permanencia no están motivados por una actividad lucrativa. +

\section{Usabilidad}

Baeza 2002 [1], la usabilidad se refiere a la capacidad de un producto de software (en este caso, una página web) para que este sea entendido, aprendido, utilizado y atractivo para el usuario (siempre que el producto sea empleado bajo las condiciones especificadas). La usabilidad tiene cinco atributos definidos: facilidad de aprendizaje, velocidad de desempeño, tasas de error por parte de los usuarios, retención sobre el tiempo, y satisfacción subjetiva.

John Cato 2001 [2], sugiere los siguientes atributos para asegurar la usabilidad de un producto:

Control.- Los usuarios deben de sentir que tienen el control por sobre la aplicación, y no al revés. Habilidades.- Los usuarios deben de sentir que el sistema apoya, complementa y realza sus habilidades y experiencia.

Privacidad.- El sistema ayuda a los usuarios a proteger su información o la de sus clientes.

En 1997 Keith Instone [9], retomó las heurísticas de usabilidad en la web de Nielsen y las adaptó específicamente para la web.

\section{Visibilidad del estado del sistema.}

El usuario siempre debe de saber exactamente qué es lo que el sistema está haciendo. En sitios web esto se puede lograr informándole al usuario dónde se encuentra, siempre. Uno de los mayores problemas para los usuarios al navegar por la red es la desorientación. Es vital asegurar una consistencia de todo el sitio web.

\section{Similitud entre el sistema y el mundo real.}

El sistema debe de hablar el lenguaje del usuario, Las frases, palabras y conceptos deben de ser familiares para el usuario. 


\section{Control por parte del usuario y libertad}

Los usuarios frecuentemente eligen funciones por error y necesitarán de "salidas de emergencia" claramente marcadas. Se debe de proveer al visitante al sitio, el contar con funciones para deshacer y rehacer las acciones que haya realizado.

\section{Consistencia y cumplimiento de estándares}

Hay que seguir las convenciones de las plataformas en las que se está desplegando el sitio web. Preferir los estilos por default de botones, barras de scroll, etc. provistas por la plataforma. El cumplimiento con las recomendaciones emitidas por el W3C (World Wide Web Consortium) referentes tecnologías web (HTML, CSS, XML,etc.) aseguran en muchos casos una portabilidad absoluta del sitio web. Se recomienda fuertemente desarrollar un plan claro en donde se defina claramente el estilo y layout del sitio web.

\section{Prevención de errores}

Aún mejor que el desarrollar buenos mensajes de error es tener un diseño cuidadoso que eviten la ocurrencia de errores. Hay que asegurarse de que las instrucciones estén escritas de una manera clara y que estás sean desplegadas de manera conveniente, evitando cualquier tipo de contaminación visual.

\section{Preferencia al reconocimiento frente a la memorización.}

Haga que los objetos, acciones y opciones sean visibles. El usuario no tiene porque recordar información de una parte de un diálogo a otra parte. Las instrucciones de uso del sistema deben de ser visibles y accesibles cuando el usuario lo considere necesario.

\section{Flexibilidad y eficiencia de uso}

Los aceleradores -invisibles para el usuario novato - pueden hacer más rápida la interacción para el usuario experto. El sistema debe de tratar eficientemente, tanto, a los usuarios expertos como inexpertos. Para lograr esto, es conveniente permitir a los usuarios que personalicen ciertas acciones frecuentes (un ejemplo de esto es la opción "1-Click" de Amazon que ofrece a los compradores habituales el pasar directamente a la confirmación de la venta, sin realizar el procedimiento estándar de facturación).

\section{Estética y diseño minimalista}

Los diálogos no deben de contener información que sea irrelevante o que rara vez sea de utilidad. Cada información extra en un diálogo, compite con unidades relevantes de información y disminuye su visibilidad relativa. Se recomienda reducir el número de imágenes al mínimo. Es altamente recomendable que la información más importante sea colocada en la parte superior de la página.

\section{Ayuda para que el usuario reconozca, diagnostique y se recupere de los errores}

Los mensajes de error deben de ser expresados en un lenguaje claro (sin ambivalencias), indicando exactamente el problema, y proveyendo constructivamente de una solución.

\section{Ayuda y documentación}

Cualquier información debe de ser fácil de buscar, y debe de estar orientada a las acciones del usuario. En cuanto un sitio web ofrezca alguna característica fuera de la norma, o ligeramente complicada, será necesario prestar ayuda y dar documentación a los usuarios.

\section{ESTADO DEL ARTE}

Según Oliveira 2010[12], los resultados de su investigación muestran que las compañías rurales tienen un conocimiento limitado sobre el uso potencial de las herramientas tecnológicas que soportan el marketing web, recomienda el incremento del uso del internet para comunicarse a nivel mundial y lograr posicionarse y obtener sostenibilidad.

Furio y Alonso 2006 [5] su trabajo se centró en el estudio de los servicios de internet a las empresas turísticas, analiza los portales turísticos; luego analiza a los actores comprometidos en el negocio como son las cadenas hoteleras, las compañías aéreas, y las agencias de viajes. Dimitrios Buhalis 2008 [3] en su trabajo analiza los estudios anteriores sobre las aplicaciones del internet en el turismo, además se proyecta sobre el desarrollo futuro del e-Turismo, que puede cambiar e 
influenciar la estructura de la industria del turismo. El mayor aporte del artículo es la revisión general sobre las investigaciones realizadas y el esfuerzo desarrollado en este campo y los retos que los investigadores en turismo se estarán enfrentando.

Según Xiaoqiu, Buhalis,y Haisan 2003 [17] en sus estudios examina como las TIC's y el internet están cambiando gradualmente la estructura de la industria del turismo en China, y la importancia de estos cambios que conducirán la industria de turismo en China. La investigación exploratoria se llevó a cabo sobre la recopilación de información de varias organizaciones turísticas como líneas aéreas, hoteles, operadores turísticos, lugares turísticos y las autoridades turísticas.

Hatem 2012 [8], menciona los factores que inciden en la adopción e implementación del E-Marketing en la industria del turismo en Egipto; los resultados indicaron que los factores internos y externos tienen un gran impacto en la adopción de E-Marketing por dichas organizaciones, que el TAM y el IDT son válidas para ilustrar el Emarketing, además los resultados refuerzan la importancia de los factores ambientales en la adopción del E-Marketing. Guerrero 2011[7], su estudio se centra en determinar los factores que promueven la utilización del E-commerce, orientados en la implementación de páginas web, como medio de negociación comercial en las PYMES del sector turístico (agencias de viaje y turismo) el distrito de San Miguel, Lima- Perú.

Sobre los estudios de Turismo publicados de 1996 a julio de 2009 [16] se refieren a los enfoques metodológicos de evaluación de sitios web. El estudio analiza la obra inicial y continúa hasta la evolución reciente de evaluación de sitios web. La investigación previa se puede dividir en cinco criterios de evaluación: contar, cálculo automático, numérico, el juicio del usuario, y métodos combinados. Las fortalezas y debilidades de cada método son examinadas. La naturaleza intensiva de la información en la industria del turismo [4], sugiere la importancia del Internet y la tecnología Web en la promoción y comercialización de los destinos. En el estudio se utiliza el modelo ampliado de Adopción Internet Comercio para evaluar el nivel de desarrollo de sitios Web en las Organizaciones Regionales de Turismo de Nueva Zelanda. El documento pone de relieve la utilidad de usar la interactividad para medir la madurez relativa de los sitios Web de turismo.

\section{METODO DE INVESTIGACION}

Lima es una ciudad que cuenta con una población de 7.605.742 habitantes, en un área de $804 \mathrm{~km} 2$, alrededor de 683 agencias de viajes. A continuación describiremos el procedimiento a seguir para determinar la usabilidad del internet. El instrumento utilizado fue un cuestionario se consideró la siguiente información:

$\checkmark$ información general (tipo de agencia, oferta de paquetes turísticos, tipo de paquete, tamaño y población objetivos).

$\checkmark$ información sobre el uso del internet (frecuencia, medios de internet, página web, problemas, capacitación del personal, servicios que ofrece la página web). El instrumento esta constituido por 16 preguntas.

El estudio se aplicó a 52 agencias de Lima Metropolitana, se obtuvo información de 14 agencias mayoristas y 38 agencias minoristas. La validación del instrumento fue sometido a juicio de expertos, fue validado por dos especialistas del área de tecnologías de información, dos administradores de agencias de turismo, jefa de instituto de investigación y especialista de estadística. Los expertos evaluaron la pertinencia, claridad y relevancia, que sirvieron para realizar los ajustes al instrumento.

\section{ANALISIS DE RESULTADOS}

\section{Agencias mayoristas}

Las agencias mayoristas consideraron que el internet es un medio importante para el desarrollo de sus actividades y es usado casi siempre tal como se aprecia en la fig. 1.

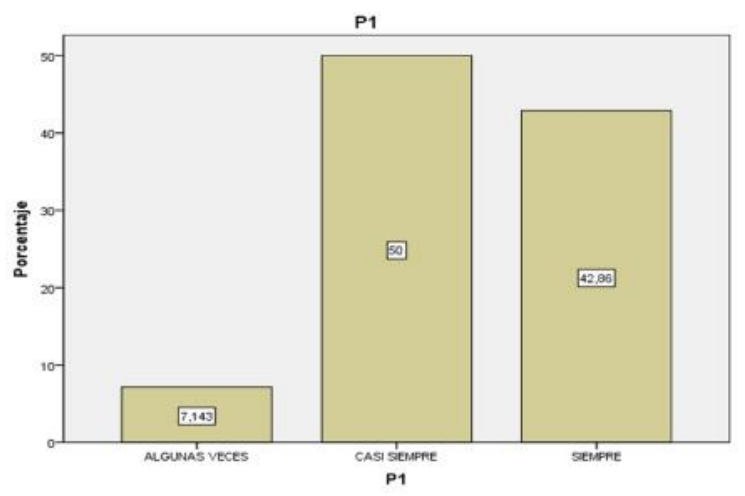

Fig. 1 Internet como herramienta de trabajo. 
Los medios de internet mas usados para captar turistas, es la página web en primer lugar, seguido de correo electronico, y luego redes sociales, según la Fig. 2.

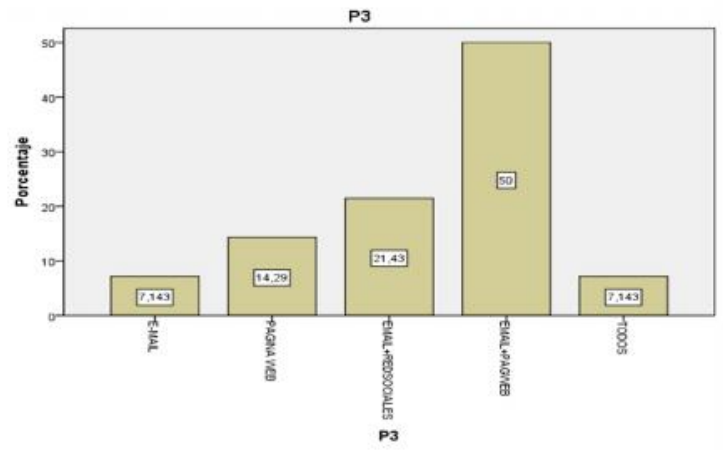

Fig. 2 Medios de internet utilizados para captar turistas.

La mayoría de las páginas web de la agencias mayoristas son informativas, algunas son interactiva con un nivel bajo, según se aprecia en la Fig. 3.

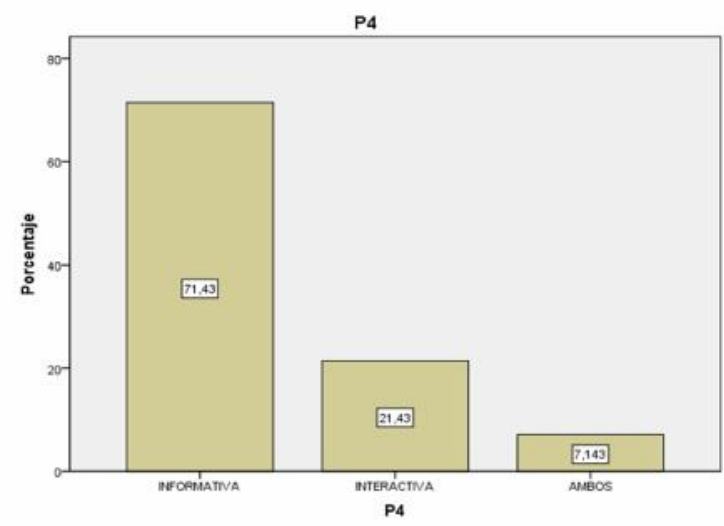

Fig. 3 Página web es informativa, interactiva.

Referente a los idiomas de las páginas web, la mayoría tiene un solo idioma que es el español, otras tiene dos idiomas español-inglés; y muy pocas tienen varios idiomas, según se muestra en la Fig. 4.

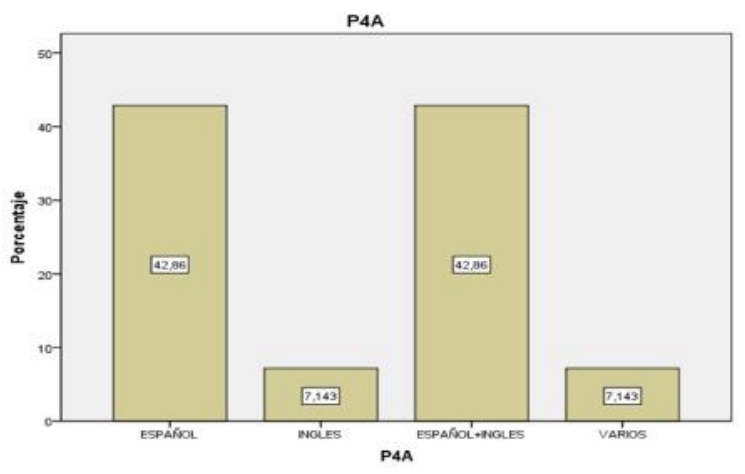

Fig. 2 Idiomas de la página web.

Sobre el tipo de turismo que realizan el turista extranjero con más frecuencia es el cultural, seguido de naturaleza, aventura y algunas combinaciones; se observa que el esotérico está siendo atractivo para un tipo de turista especial, según se observa en la Fig. 5.

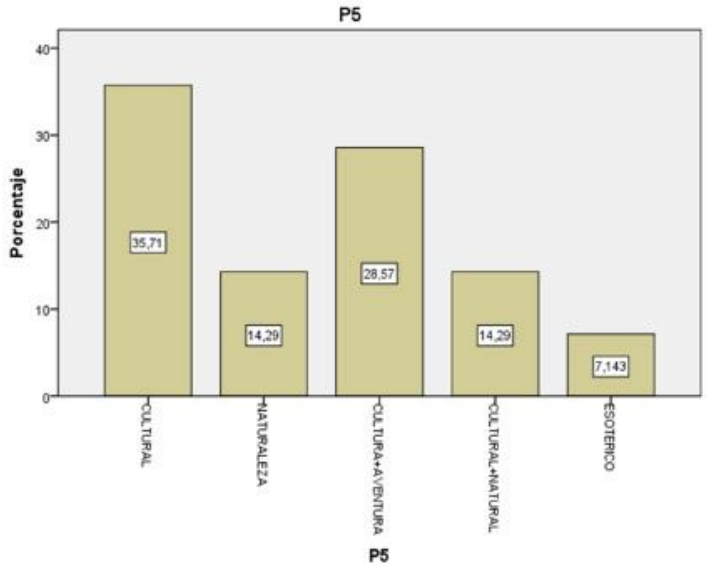

Fig. 3 Tipo de turismo que realizan los turistas extranjeros.

El problema más significativo que afecta el uso de internet sobre aplicaciones de negocios electrónicos, en primer lugar, es costos, debido a que aplicaciones en internet son caras, luego tecnológico, y en tercer lugar está el aspecto personal y procedimientos, según la Fig. 6. 


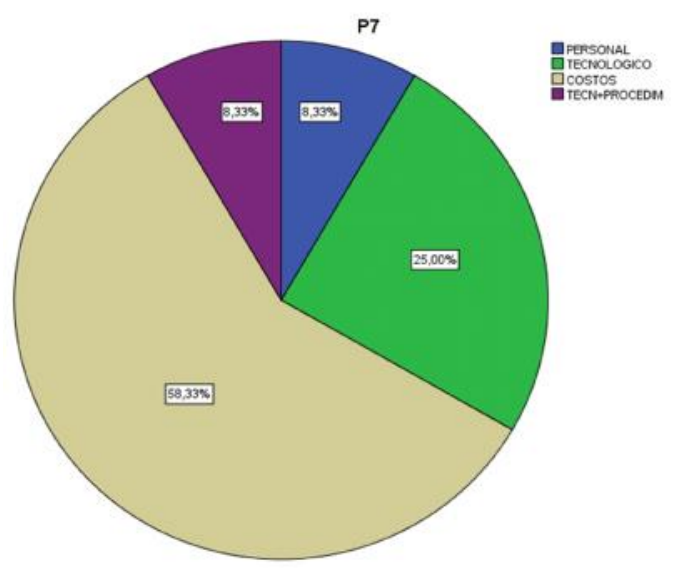

Fig. 4 Problemas que afectan el uso del Internet para captar turistas.

Los servicios en linea que ofrece las paginas web de las agencias mayoristas, es el registrar pedidos $\mathrm{y}$ responder e-mail, otras agencias no tienen ningun servicio en linea. Ver Fig. 7.

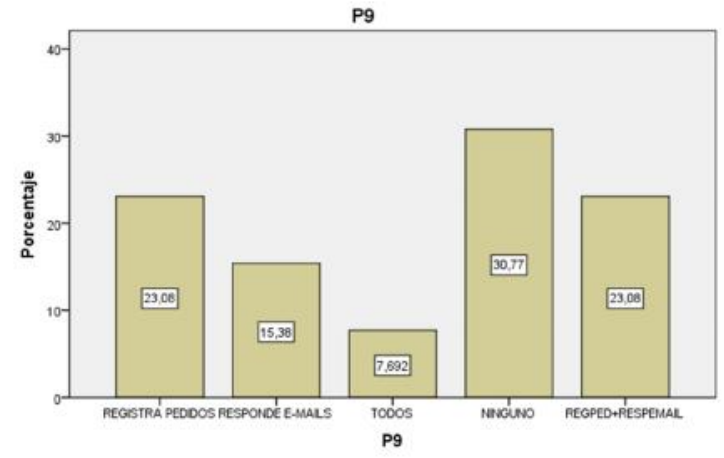

Fig. 5 Servicios en línea que ofrece la página web.

Sobre los servicios de comercio como: pago electrónico, canasta de pedidos; observamos que un gran porcentaje no cuentan con ningún de este servicios; el pago electrónico y canasta de pedidos es muy bajo solo algunas agencias ofrecen estos servicios, según la Fig. 8.

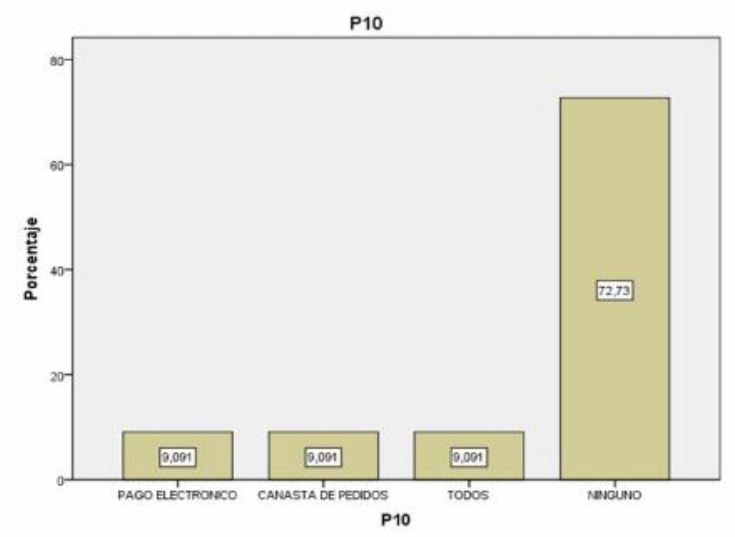

Fig. 6 Servicios en línea que la web ofrece a los turistas.

El personal que trabaja en las agencias tienen un nivel de capacitación básico e intermedio en el manejo del Internet, y muy pocas con un nivel avanzado. Ver Fig. 9.

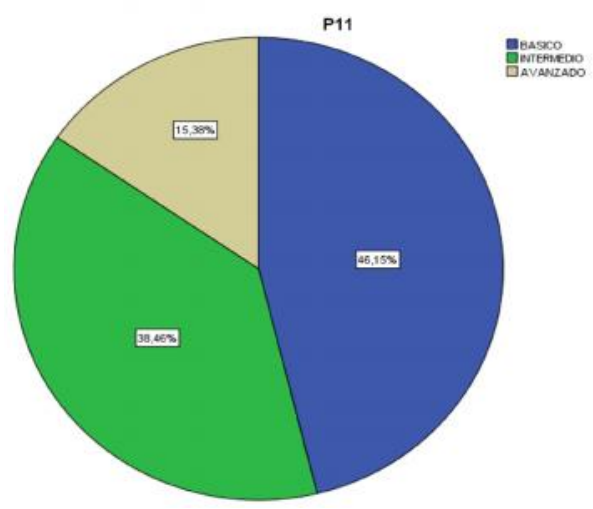

Fig. 7 Capacitación del personal en el manejo del Internet.

\section{Agencias minoristas}

Las agencias minoristas consideraron que el internet es un medio importante para el desarrollo de sus actividades y es usado casi siempre como se aprecia en la fig. 10. 


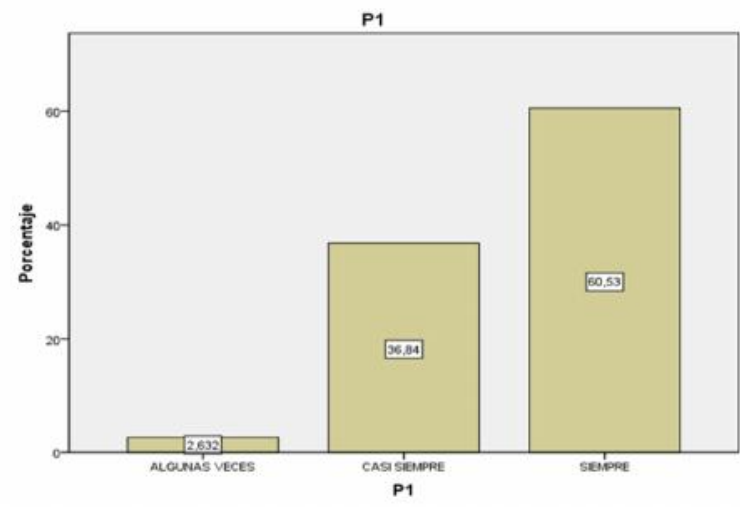

Fig. 10 Internet como herramienta de trabajo.

Los medios de internet mas usados para captar turistas, es la pagina web en primer lugar, seguido de correo electronico, chat, y luego redes sociales, según la Fig. 11.

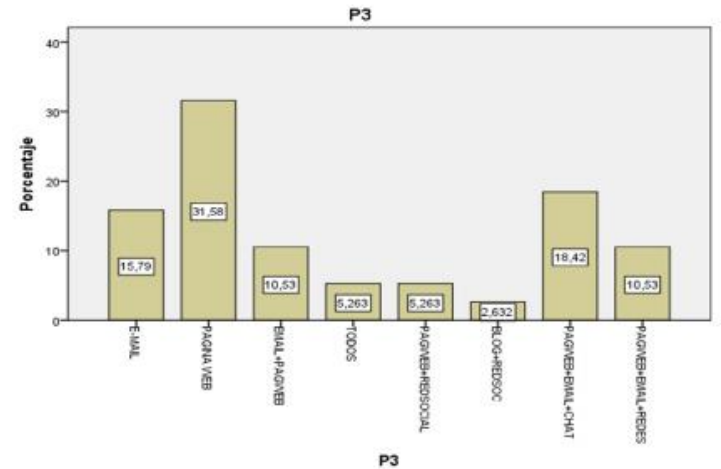

Fig. 8 Medios de Internet utilizados para captar turistas.

La mayoría de las páginas web de la agencias minoristas son informativas, algunas son interactivas con un nivel muy bajo según se aprecia en la Fig. 12.

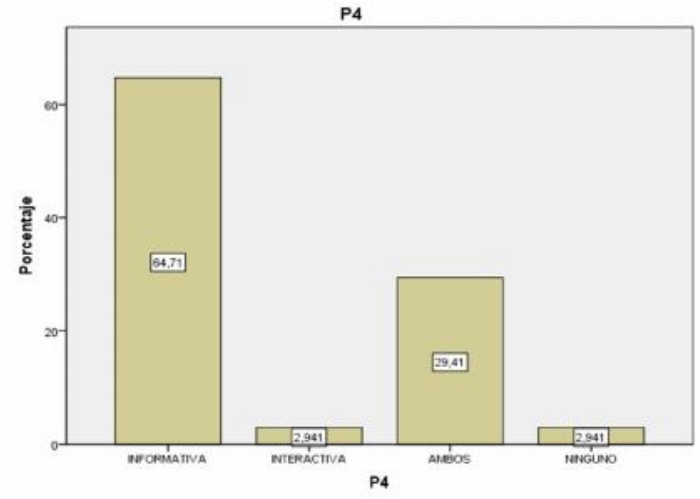

Fig. 9 Pagina web es informativa, interactiva.
Referente a los idiomas de las páginas web, la mayoría tiene un solo idioma que es el español, otras tiene dos idiomas español-inglés; y muy pocas tienen varios idiomas, según se muestra en la Fig.13.

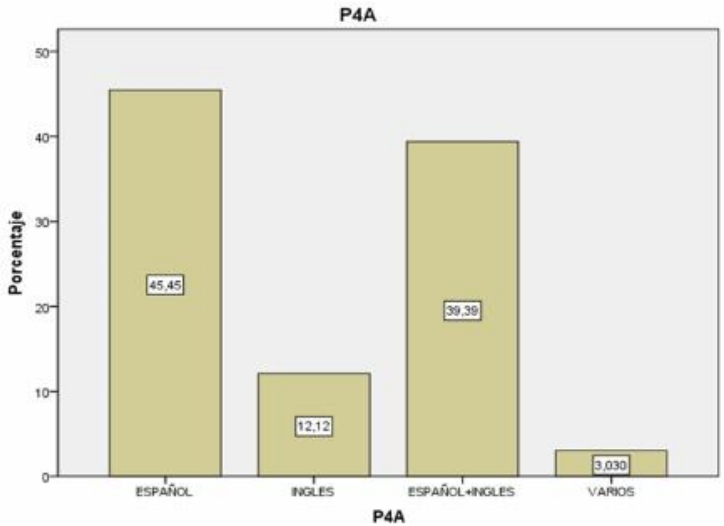

Fig. 10 Idiomas de la página web

Sobre el tipo de turismo que realizan el turista extranjero con más frecuencia es el cultural, seguido de naturaleza, aventura y algunas combinaciones; se observa que el esotérico está siendo atractivo para un tipo de turista especial, según se observa en la Fig. 14.

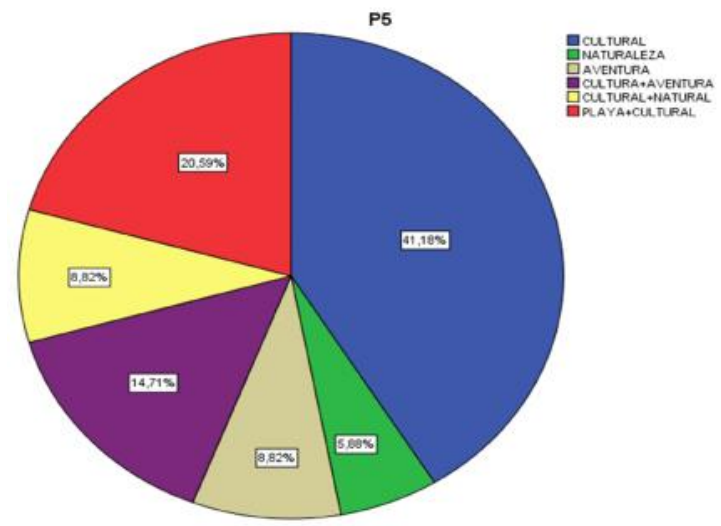

Fig. 11 Tipo de turismo que realizan los turistas extranjeros.

El problema más significativo que afecta el uso de internet sobre aplicaciones de negocios electrónicos en primer lugar es procedimientos, luego personal-tecnológico, y en tercer lugar está el aspecto de costos, según la Fig. 15. 


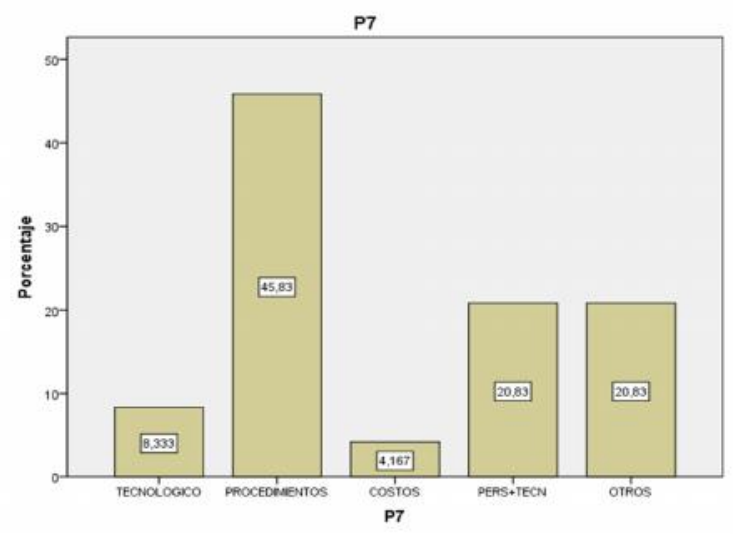

Fig. 12 Problemas que afectan el uso de internet para captar turistas.

Los servicios en línea que ofrece las páginas web de las agencias minoristas son: facilita seguimiento de solicitudes, registra pedidos y responde e-mail, $\mathrm{y}$ algunos ofrecen cotizaciones, y otras agencias no tienen ningún servicio en línea. Ver Fig. 16.

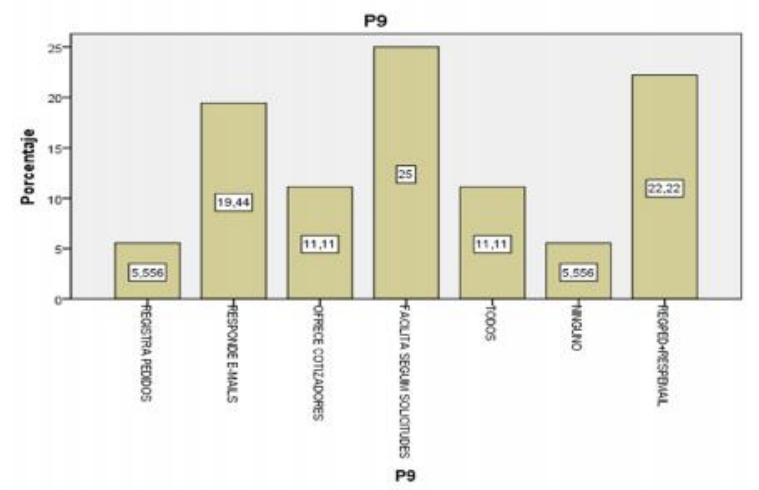

Fig. 13 Servicios en línea que ofrecen la pág. Web.

Sobre los servicios de comercio electrónico las agencias minoristas ofrecen canastas de pedidos, observamos que un gran porcentaje no cuentan con ninguno de estos servicios; y el pago electrónico es muy bajo solo algunas agencias ofrecen estos servicios, según la Fig. 17

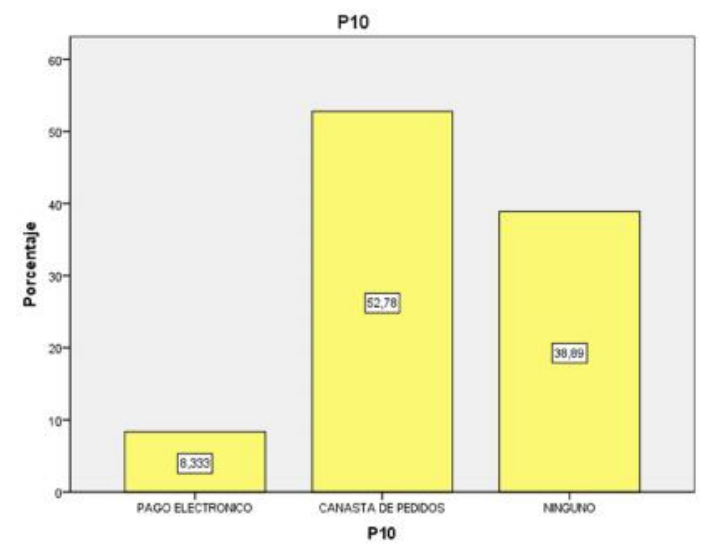

Fig. 14 Servicio de comercio electrónico.

El personal que trabaja en las agencias minoristas tienen un nivel de capacitación intermedia, y básico en el manejo del internet. Ver Fig. 18.

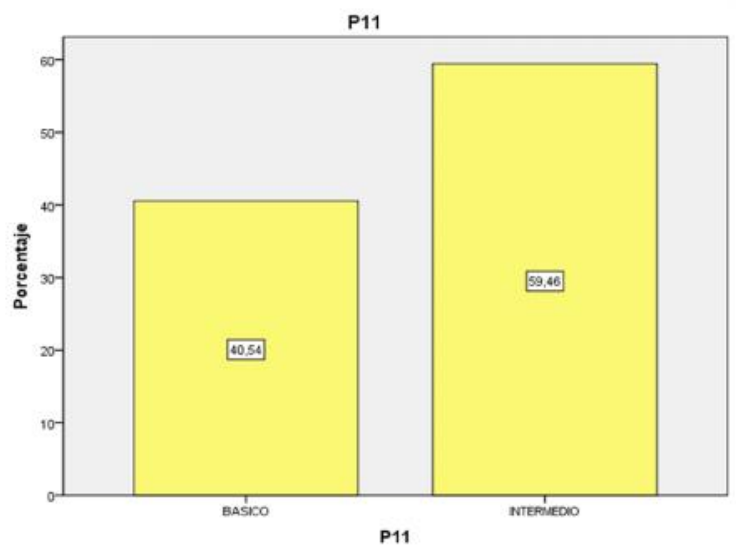

Fig. 15 Capacitación del personal.

\section{CONCLUSIONES}

Tanto las agencias mayoristas como las agencias minoristas consideran importante el uso del internet para el desarrollo de sus actividades.

Que los medios de Internet más usados en las actividades de captación de turistas por las agencias mayoristas y minoristas son el primer lugar, la Página web y el E-mail; en segundo lugar están, el chat, y las redes sociales. 
Las páginas web de las agencias mayoristas y minoristas son más informativas que interactivas, siendo la función "interactiva" más fuerte en las agencias mayoristas que en los minoristas.

Los idiomas que tienen la mayoría de las páginas web de la agencia mayoristas y las minoristas, es el idioma español, luego español + ingles, muy pocos tienen varios idiomas.

Sobre el tipo de turismo más frecuente que realizan los turistas extranjeros, se observa que el "cultural" está en primer lugar, seguido de "cultural + aventura", hay otros tipos de turismo que son muy atractivo es el "esotérico o místico" y el "gastronómico".

Los problemas que más afectan el uso del Internet para captar turistas son: el tecnológico, costos y procedimientos; ligeramente son los de personal. Observándose un nivel alto sobre "costos" en las agencias mayoristas y el de "procedimientos" en las minoristas.

Los tipos de servicio en línea que ofrece la página web de las agencias son: en primer lugar registrar pedidos, luego responder email, y algunos ofrecen cotizaciones de servicios que son lo más frecuente.

Las páginas web de las agencias respecto el pago electrónico no es muy usado, las agencias minoristas usan con más frecuencia la canasta de pedido, y las agencias mayoristas mostraron un alto porcentaje sobre ninguno.

El personal que trabaja en las agencias de turismo está bien preparado o bien capacitado en el uso del internet con buen nivel intermedio.

\section{REFERENCIAS}

1. Baeza, R., "Ubicuidad y Usabilidad en la Web". (2002).

2. Cato, J., "User-centered web design". (2001).

3. Dimitrios, B., "Progress in Information technology and tourism management", 20 years on and 10 years after the Internet. (2008).
4. Bill, D., "Evaluating the use of the web for tourism marketing: a case study from", New Zeland. (2002).

5. Furio, A., "Internet y sus aplicaciones al sector turístico". (2006).

6. Gonzales, Julca., "Tecnologías de Información aplicables al sector turismo en el Perú”, UNMSM, Tesis, Lima-Perú. (2005).

7. Guerrero, J., "Factores que promueven la utilización del E-commerce como medio de negociación comercial en las agencias de viaje y turismo en el distrito de San Miguel". Revista de Ciencias Empresariales de la Universidad San Martin de Porres. Vol. 2. Num. 2. pp. 53-59. (2011).

8. Hatem El-Gohary., "Factors affecting EMarketing adoption and implementation intourism firms". (2012).

9. Instone, K., "Site usability Heuristics for the web". (1997).

10. Lacramioara, L., "Las Nuevas tecnologías en el turismo". (2007).

11. Mendia, Herrero., "La guía turística móvil". TURITEC-2006 - (2006).

12. Oliveira Duarte., "Use and Perception of the Internet as a Marketing Tool to promote Rural Tourism". (2010).

13. Organización Mundial Turismo. (2008).

14. OTP-BADATUR, INEI. "Observatorio turístico del Peru". (2012).

15. Pablo, R., "Las nuevas tecnologías aplicadas al turismo. Madrid, España. (2004).

16. Law, R., "Progress in tourism management: a review of website evaluation in tourism research". (2010).

17. Xiaoqiu, Ma., "ICTs and internet adoption in China`s tourism industry". (2003).

18. Hunziker, W., - Krapf, K., "Fundamentos de la Teoría General del Turismo". 1942.

Correspondencia: merynoemi0601@yahoo.com

Recepción de originales: enero 2013

Aceptación de originales: mayo 2013 